\title{
Performance of Multistage Interference Cancellers in Space-Time Coded CDMA Systems
}

\author{
M. M. Alam and A. Chockalingam \\ Department of Electrical Communication Engineering \\ Indian Institute of Science, Bangalore 560012. INDIA
}

\begin{abstract}
In this paper, we investigate the bit error performance of multistage interference cancellers in a $K$-user asynchronous/synchronous space-time coded CDMA system under near-far conditions. We consider orthogonal space-time block codes with $M, 1 \leq M \leq 8$ transmit antennas. We develop a generalized discrete time vector. model for the received signal at the output of the matched filters, and use this model to evaluate the bit error performance of multistage interference cancellers (ICs). Our performance results show that a) when accurate fade amplitude and timing estimates are available, the ICs are Iess near-far resistant at low near-far ratios (NFRs) compared to that at high NFRs, due to the unreliability of the interfering users' data estimates in the initial stages of the IC at low NFRs, and b) in the high NFR region, the near-far resistance is degraded due to inaccuracies in fade amplitude estimates. This indicates that that some amount of transmit power control will be desired in ICs to counter the loss of near-far resistance due to the above reasons.
\end{abstract}

Keywords: Space-time codes, CDMA, interference cancellation

\section{INTRODUCTION}

The use of space-time coded transmission in multiuser scenarios is being increasingly adopted in communication systems design (e.g., in $3 \mathrm{G}$ cellular systems). Space-time coding with multiple transmit antennas provides transmit diversity benefits and can achieve high data rates on wireless fading channels [1]-[4]. Also, interference rejection schemes using multiuser detection techniques can significantly enhance the receiver performance and increase the capacity of code division multiple access (CDMA) systems. Multiuser detection techniques have been extensively studied in literature, mainly for single transmit antenna systems [5]. Our focus in this paper is the investigation of interference canceling multiuser receiver structures and their performances in a space-time coded multiuser scenario. Recently reported works in the area of spacetime multiuser detection include [6]-[8]. In [6], a combined interference suppression and maximum likelihood (ML) decoding scheme for space-time block codes to suppress the interference from other co-channel users in a TDMA system was presented. This system however required the number of receive antennas to be greater the number of users in the system. In [7], Huang et al considered ML space-time multiuser detection in a CDMA system. In [8], Reynolds et al considered the performance of blind adaptive space-time multiuser detection for a two transmit antenna scheme.

In this paper, we investigate the bit error performance of multistage interference cancellation techniques for a space-time

\footnotetext{
This work was supported in part by the Swamajayanti Fellowship from the Department of Science and Technology, Government of India, New Delhj, under scheme Ref: No. 6/3/2002-S.F
}

coded CDMA system. We first develop a generalized discretetime vector model for the received signal at the output of the matched filters in a $K$-user synchronous/asynchronous CDMA system. We construct this vector model for orthogonal space-time block codes with $M, 1 \leq M \leq 8$ transmit antennas. Using this model, we evaluate the bit error performance of multistage interference cancellers (ICs) under near-far conditions. Our performance results show that a) when accurate fade amplitude and timing estimates are available, the ICs are less near-far resistant at low near-far ratios (NFRs) compared to that at high NFRs, due to the unreliability of the interfering users' data estimates in the initial stages of the IC at low NFRs, and b) in the high NFR region, the near-far resistance is degraded due to inaccuracies in fade amplitude estimates. This indicates that that some amount of transmit power control will be desired in ICs to counter the above loss of near-far resistance.

The rest of the paper is organized as follows. In Section II, we derive the vector model for the received signal._In.Section III, we develop the structure of the interference cancellers using the vector model. Section IV presents the bit error performance results, and Section V gives the conclusions.

\section{II! SYSTEM MODEL}

We consider a $K$-user asynchronous CDMA systém with $M$ transmit antennas per user. Users transmit frames of $(2 L+1)$ data blocks with $Q$ bits per data block. Let $b_{i q}(m), i=$ $1,2, \ldots, K, q=1,2, \ldots, Q, m=-L, \ldots, 0, \ldots, L$, be the the $q^{\text {th }}$ bit of the $m^{\text {th }}$ block of the $i^{\text {th }}$ user, transmitted in a time interval of length $T$. The bits in a data block are mapped onto the $M$ transmit antennas using real orthogonal spacetime block codes (STBC). We assume that the channel fading is quasi-static and the quasi-static interval is $Q T$ time units, where $Q=2^{r}, r$ being the smallest integer satisfying $Q \geq M$ [3]. For square real orthogonal STBC with $M=Q=8$, the transmission matrix $\mathbf{B}$ is given by [4]

$$
\mathbf{B}=\left[\begin{array}{cccccccc}
b_{1} & b_{2} & b_{3} & b_{4} & b_{3} & b_{6} & b_{7} & b_{8} \\
b_{2} & -b_{1} & b_{4} & -b_{9} & b_{6} & -b_{5} & -b_{8} & b_{7} \\
b_{3} & -b_{4} & -b_{1} & b_{2} & b_{7} & b_{8} & -b_{5} & -b_{6} \\
b_{4} & b_{3} & -b_{2} & -b_{1} & b_{8} & -b_{7} & b_{6} & -b_{5} \\
b_{5} & -b_{6} & -b_{7} & -b_{8} & -b_{1} & b_{2} & b_{3} & b_{4} \\
b_{6} & b_{5} & -b_{8} & b_{7} & -b_{2} & -b_{1} & -b_{4} & b_{3} \\
b_{7} & b_{8} & b_{5} & -b_{6} & -b_{3} & b_{4} & -b_{1} & -b_{2} \\
b_{8} & -b_{7} & b_{8} & b_{5} & -b_{4} & -b_{3} & b_{2} & -b_{1}
\end{array}\right]
$$

In the above transmission matrix, the columns represent the transmit antenna index and the rows represent the bit interval index. The transmission matrix $\mathbf{B}$ for other real orthogonal designs for $M, Q<8$ can be obtained to be the upper leftmost 


\section{TENCON $2003 / 138$}

submatrix of $\mathbf{B}$ of order $Q \times M$. For example, $M=Q=2$ the transmission matrix $B$ can be obtained to be

$$
\mathbf{B}=\left[\begin{array}{cc}
b_{1} & b_{2} \\
b_{2} & -b_{1}
\end{array}\right] \text {. }
$$

We are interested in developing a vector model for the received signal. In the following, we first illustrate the construction of the model using $M=Q=2$ as an example, and then state the generalization of the model for any $M \leq 8$.

For the $M=Q=2$ system, the received signal on a receive antenna can be written using (2) as

$$
\begin{aligned}
& y(t)=y_{1}(t)+y_{2}(t)+z\langle t\rangle, \\
& y_{1}(t)=\sum_{m=-L}^{L} \sum_{i=1}^{K} A_{i 1} h_{i 1}(m)\left\{b_{i 1}(m) s_{i 1}^{(m)}+b_{i 2}(m) s_{i 2}^{(m)}\right\}, \\
& y_{2}(t)=\sum_{m=-L}^{L} \sum_{i=1}^{K} A_{i 2} h_{i 2}(m)\left\{b_{i 2}(m) s_{i 1}^{(m)}-b_{i 1}(m) s_{i 2}^{(m)}\right\} .
\end{aligned}
$$

In the above, $\left.t_{p}\right), p \in\{1,2$ is the signal component due to the $p^{\text {th }}$ transmit antenna, $A_{i p}$ is the transmit amplitude on the $p^{\text {th }}$ transmit antenna of the $i^{t h}$ user, $h_{i p}(m)$ is the complex channel gain for the $m^{\text {th }}$ block from the $p^{\text {th }}$ transmit antenna of the $i^{t h}$ user, and $s_{i q}^{(m)}$ represents the signature waveform of the $i^{\text {th }}$ user for the $q^{\text {th }}$ bit in a block, $q \in\{\}, 2$, given by

$$
s_{i q}^{(m)}=s_{i}\left(t-m Q T-\overline{q-1} T-\tau_{i}\right),
$$

where $s_{i}(t)$ is a unit energy waveform time limited in the interval $\left[0, T\right.$, and $\tau_{i}$ is the random time delay of the $i^{t h}$ user which we assume to be independent of the transmitting antenna and to satisfy $\tau_{i} \geq T$ "for $j>i$. Also, $z(t)$ is a zero mean complex Gaussian noise process with variance $2 \sigma^{2}$.

The demodulator on each receive antenna uses a bank of $K$ matched filters, each matched to a different user signature waveform. With the timing of all the users, $\tau_{j}, j=1,2, \ldots, K$ being known, the received signal at the output of the matched filters can be written as

$$
y_{j q}(n)=\int_{n Q T+\tau_{j}}^{(n+1) Q T+\tau_{j}} y(t) s_{j q}\left(t-n Q T-\tau_{j}\right) d t,
$$

where $j=1,2, \ldots, K, q=1,2, \ldots, Q$ and $n=-L, \ldots, 0, \ldots, L$. We have two cross-correlations between every pair of signature waveforms that depend on the time offset $\tau$ between the users. If $j<k, 1 \leq j, k \leq K$, we have

$\rho_{j k}(\tau)=\int_{\tau}^{T} s_{j}(t) s_{k}(t-\tau) d t, \rho_{k j}(\tau)=\int_{0}^{\tau} s_{j}(t) s_{k}(t+T-\tau) d t$,

where $\tau \quad F_{j}\left[\quad q_{j} T\right.$. Using (8), we define the following $K K$ matrices:

$\mathbf{R}[0]=\left[\begin{array}{cccc}1 & \rho_{12}\left(r_{2}-r_{1}\right) & \ldots & \rho_{1 K}\left(r_{K}-r_{1}\right) \\ \rho_{12}\left(\tau_{2}-r_{1}\right) & \rho_{2 K}\left(r_{K}=r_{11}\right. \\ \vdots & \vdots & \vdots & \vdots \\ \rho_{1 K}\left(r_{K}-r_{1}\right) & \rho_{2 K}\left(r_{K}-r_{21}\right. & \cdots & 1\end{array}\right]$.

$\mathbf{R}[1]=\left[\begin{array}{ccccc}0 & \rho_{21}\left(r_{2}-r_{1}\right) & \rho_{332}\left(r_{3}-r_{1}\right) & \ldots & { }_{\rho_{32}\left(r_{3}-r_{2}\right)} \\ 0 & \rho_{K 1}\left(\tau_{K}-r_{1}\right) & \cdots & \rho_{K 2}\left(\tau_{K}-r_{2}\right) \\ \vdots & \vdots & \vdots & \vdots & \vdots \\ 0 & 0 & \cdots & 0 & \rho_{K \overline{K-1}}\left(r_{K}-\tau_{K-1}\right) \\ 0 & 0 & 0 & 0 & 0\end{array}\right]$
It is noted that, when we consider synchronous CDMA, $\mathbf{R}[1]=\mathbf{0}$. The following matrices would help us write (7) in vector notation:

$$
\begin{array}{r}
\mathbf{y}_{q}(n)=\left[y_{1_{q}}(n), \ldots, y_{K_{q}}(n)\right]^{T}, \\
\mathbf{y}(n)=\left[\mathbf{y}_{1}^{T}(n), \mathbf{y}_{2}^{T}(n), \ldots, \mathbf{y}_{Q}^{T}(n)\right]^{T}, \\
\mathbf{y}=\left[\mathbf{y}^{T}(-L), \ldots, \mathbf{y}^{T}(0), \ldots, \mathbf{y}^{T}(L)\right]^{T}, \\
\mathbf{H}_{q}(n)=\operatorname{diag}\left[A_{1 q} h_{1 q}(n), \ldots, A_{K_{q}} h_{K q}(n)\right], \\
\mathbf{H}(n)=\left[\begin{array}{rr}
\mathbf{H}_{1}(n) & \mathbf{H}_{2}(n) \\
-\mathbf{H}_{2}(n) & \mathbf{H}_{1}(n)
\end{array}\right], \\
\mathbf{H}=\operatorname{diag}[\mathbf{H}(-L), \ldots, \mathbf{H}(0), \ldots, \mathbf{H}(L)], \\
\mathbf{b}_{q}(n)=\left[b_{1 q}(n), \ldots, b_{K_{q}}(n)\right]^{T}, \\
\mathbf{b}(n)=\left[\mathbf{b}_{1}^{T}(n), \ldots, \mathbf{b}_{Q}^{T}(n)\right]^{T}, \\
\mathbf{b}=\left[\mathbf{b}^{T}(-L), \ldots, \mathbf{b}^{T}(0), \ldots, \mathbf{b}^{T}(L)\right] .
\end{array}
$$

Using the above definitions, it can be shown that

$$
\begin{aligned}
& \mathbf{y}=\mathbf{C H b}+\mathbf{z}, \\
& \mathbf{C}=\left[\begin{array}{ccccc}
\mathbf{R}[0] & \mathbf{R}^{T}[1] & 0 & \ldots & \ldots \\
\mathbf{R}[1] & \mathbf{R}[0] & \mathbf{R}^{T}[1] & 0 & \ldots \\
\mathbf{0} & \mathbf{R}[1] & \mathbf{R}[0] & \mathbf{R}^{T}[1] & \ldots \\
\vdots & \vdots & \vdots & \vdots & \vdots \\
\mathbf{0} & \cdots & 0 & \mathbf{R}[1] & \mathbf{R}[0]
\end{array}\right] . \\
& E\left[\mathbf{z z}^{H}\right]=2 \sigma^{2} \mathbf{C},
\end{aligned}
$$

where $\mathbf{z}^{H}$ denotes the Hermitian of the complex vector $\mathbf{z}$.

The vector model in (20) can be valid for other values of $M$, provided the matrices, $\mathbf{H}(n)$ (of order $Q K \times Q K$ ) are defined appropriately. For example, if $M=Q=8$,

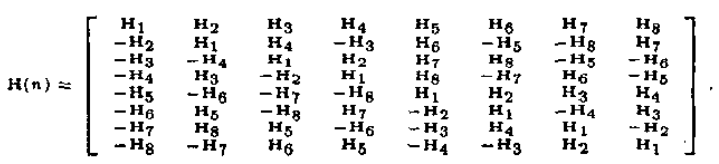

where the indices $n$ for the elements $\mathbf{H}_{q}, q=1,2, \ldots, Q$, have been omitted for convenience. It is noted that the structure of the square matrix $\mathbf{C}$ (of order $Q K(2 L+1)$ ) remains same. For values of $M$ and $Q$ other than $8, \mathbf{H}(n)$ is given by the upper leftmost submatrix of order $Q K \times Q K$ in the above Eqn. For the case of $M \notin\{4,2,, \beta, M<Q$. Therefore, only the elements $\mathbf{H}_{q}(n), q=1,2, \ldots, M$, are non-zero, i.e., $\mathbf{H}_{q}(n)=\mathbf{0}$ for $M<q \leq Q$.

To summarize, (20) gives the desired discrete-time vector model for the received signal at the output of the matched fil-

ter. The square matrix $\mathbf{C}$ contains the correlation coefficients between the signature waveforms of the users and is called the correlation matrix. The matrix $\mathbf{H}$ is the channel matrix and $\mathbf{z}$ is a zero mean complex Gaussian vector with covariance matrix given by (22).

\section{INTERFERENCE CANCELlation}

A direct consequence of the orthogonality of the STBC used for transmission is [3]

("Re $e\left(\mathbf{H}^{H} \mathbf{H}\right)=\operatorname{diag}\left[\operatorname{Re}\left[\mathbf{H}^{H}(-L) \mathbf{H}(-L)\right], \ldots, \operatorname{Re}\left[\mathbf{H}^{H}(L) \mathbf{H}(L)\right]\right]$, $\operatorname{Re}\left[\mathbf{H}^{H}(n) \mathbf{H}(n)\right]=\operatorname{diag}\left[\left(\mathbf{P}_{1}(n), \ldots, \mathbf{P}_{Q}(n)\right]\right.$ 


\section{Communications-1/139}

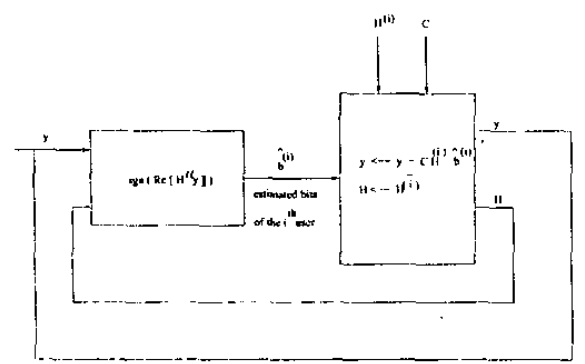

Fig. 1. Structure of the SIC

$$
\begin{array}{r}
\boldsymbol{P}_{q}(n)=\operatorname{diag}\left[\sum_{p=1}^{M} A_{1 p}^{2}\left|h_{1 p}(n)\right|^{2}, \ldots, \sum_{p=1}^{M} A_{K_{p}}^{2}\left|h_{K_{p}}(n)\right|^{2}\right], \\
q=1,2, \ldots, Q .
\end{array}
$$

The above identity facilitates coherent demodulation of the received signal using the vector model derived in the previous section. We first illustrate two suboptimum linear detectors, namely, the conventional matched filter (MF) detector and the decorrelating (DC) detector, which will be used as first stages in the multistage interference cancellers to be presented later. We assume one receive antenna throughout (although it is easy to extend the model to multiple receive antennas). We also assume perfect knowledge of the channel matrix $\mathbf{H}$ and the correlation matrix $\mathbf{C}$. The matched filter outputs at the receive antenna is given by (20).

An estimate of $\mathbf{b}$, obtained using the conventional MF detector (which does not take into account the multiple access interference via the the matrix $\mathbf{C}$ ), is given by

$$
\begin{aligned}
& \hat{\mathbf{b}}=\operatorname{sgn}\left[\operatorname{Re}\left(\mathbf{H}^{H} \mathbf{y}\right)\right] \\
& =\operatorname{sgn}\left[\operatorname{Re}\left(\mathbf{H}^{H} \mathbf{H}\right) \mathbf{b}+\operatorname{Re}\left(\mathbf{H}^{H}(\mathbf{C}-\mathbf{I}) \mathbf{H}\right) \mathbf{b}+\operatorname{Re}\left(\mathbf{H}^{H} \mathbf{z}\right)\right] .
\end{aligned}
$$

The first term in the above equation represents the desired signal, whereas the second and the last term represent the MAI and noise, respectively. The decorrelating (DC) detector can be realized by rewriting $(20)$ as

$$
\begin{aligned}
& \mathbf{u}=\mathbf{R} \mathbf{b}+\mathbf{n} \\
& \mathbf{u}=\operatorname{Re}\left(\mathbf{H}^{H} \mathbf{y}\right), \mathbf{R}=\operatorname{Re}\left(\mathbf{H}^{H} \mathbf{C H}\right) \mathbf{n}=\operatorname{Re}\left(\mathbf{H}^{H} \mathbf{z}\right) .
\end{aligned}
$$

An estimate of $\mathbf{b}$, obtained using the decorrelating detector (which suppresses the MAI), is given by

$$
\hat{\mathbf{b}}=\operatorname{sgn}\left[\mathbf{R}^{-1} \mathbf{u}\right]=\operatorname{sgn}\left[\mathbf{b}+\mathbf{R}^{-1} \mathbf{n}\right] \text {. }
$$

Now, consider the interference cancellation using successive interference canceller (SIC) and parallel interference canceller (PIC) [9],[10]. The following definitions will be useful in developing the structure of the SIC/PIC for the considered system:

- $\hat{\mathbf{b}}^{(i)}$ : is the estimated vector $\mathbf{b}$ with elements of the $i^{t h}$ user only being non-zero

- $\hat{\mathbf{b}}^{(\bar{i})}:$ is the estimated vector $\mathbf{b}$ with elements of the $i^{\text {th }}$ user being zero

- $\mathbf{H}^{(i)}$ : is the channel matrix with rows of the $i^{\text {th }}$ user only being non-zero

- $\mathbf{H}^{(\bar{i})}$ : is the channel matrix with rows of the $i^{\text {th }}$ user being zero.

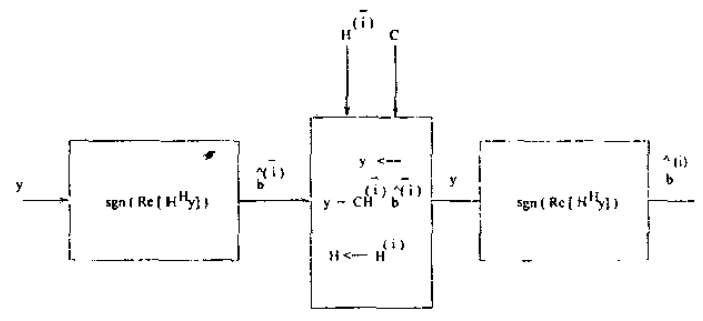

Fig. 2. Structure of the PIC

\section{Serial Interference Canceller}

For successive interference cancellation, we start with an initial estimate for vector $\mathbf{b}$ given by one of the linear detectors above (i.e., (26) or (29)). Then, we decompose $\hat{\mathbf{b}}$ and $\mathbf{H}$ as follows:

$$
\begin{aligned}
\hat{\mathbf{b}} & =\hat{\mathbf{b}}^{(i)}+\hat{\mathbf{b}}^{(\hat{i})}, \\
\mathbf{H} & =\mathbf{H}^{(i)}+\mathbf{H}^{(\bar{i})},
\end{aligned}
$$

where the $i^{t h}$ user is the strongest user. The elements of the $i^{\text {th }}$ user in $\hat{\mathbf{b}}^{(i)}$ is taken as the final estimate for the bits of the $i^{t h}$ user. Then using $\hat{\mathbf{b}}^{(i)}$, we regenerate and cancel the signal component due to the $i^{t h}$ user (i.e., the strongest user) from the signal vector $\mathbf{y}$ and modify the channel matrix $\mathbf{H}$ as follows:

$$
\begin{gathered}
\mathbf{y} \leftarrow \mathbf{y}-\mathbf{C H}^{(i)} \hat{\mathbf{b}}^{(i)}, \\
\mathbf{H} \leftarrow \mathbf{H}^{(i)} .
\end{gathered}
$$

The resulting signal vector $\mathbf{y}$ and the channel matrix $\mathbf{H}$ is then used in (26) or (29), and the above process is repeated from (30) through (33) to cancel the interference from the next strongest user. We continue the process until the bits of all the users are decoded.

\section{Parallel Interference Canceller}

For parallel interference cancellation also, we start with an initial estimate for vector $\mathbf{b}$ given by $(26)$ or $(29)$. Then we decompose $\hat{\mathbf{b}}$ and $\mathbf{H}$ as in (30) and (31), respectively. The $i^{\text {th }}$ user in this case is the desired user. Using $b^{(i)}$ as an estimate of the other user bits, we regenerate and cancel the other user interference from the signal vector $y$ and then estimate the bits of the $i^{\text {th }}$ user (i.e., the desired user) as follows:

$$
\begin{gathered}
\mathbf{y} \leftarrow \mathbf{y}-\mathbf{C H}^{(i)} \hat{\mathbf{b}}^{(\bar{i})}, \\
\hat{\mathbf{b}}^{(i)} \leftarrow \operatorname{sgn}\left[\operatorname{Re}\left(\mathbf{H}^{(i) H}, \mathbf{y}\right)\right] .
\end{gathered}
$$

We take the elements of the $i^{\text {th }}$ user in $\hat{\mathbf{b}}^{(i)}$ as an estimate for the bits of the $i^{\text {th }}$ user. A similar procedure is followed for all other users and the final estimate of the vector $\mathbf{b}$ obtained can again be used as an initial estimate for the next stage, and the overall process can be repeated.

\section{RESULTS AND DISCUSSION}

We simulated the model developed above and evaluated the bit error performance of the interference cancellation techniques under near-far conditions. A synchronous space-time coded CDMA system with two users $(K=2)$ using two transmit antennas per user $(M=2)$ is considered. The values of the 


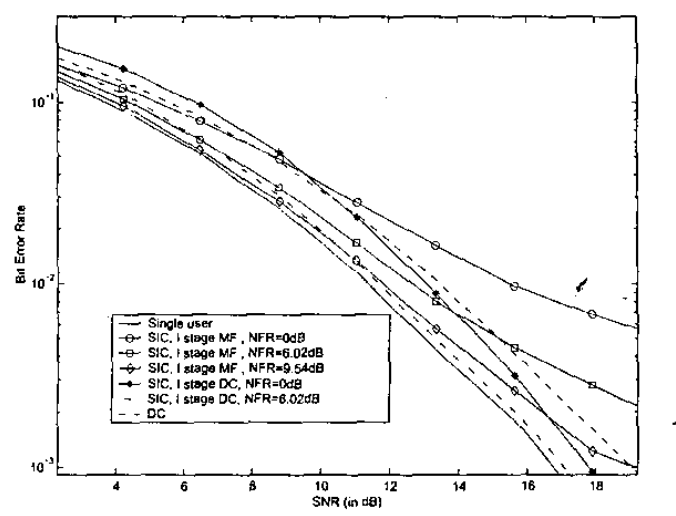

Fig. 3. BER performance of SIC for user 1 as a function of SNR in a 2-user space-time coded CDMA system. $M=2, \rho=0.8$. Perfect fade amplitude estimates.

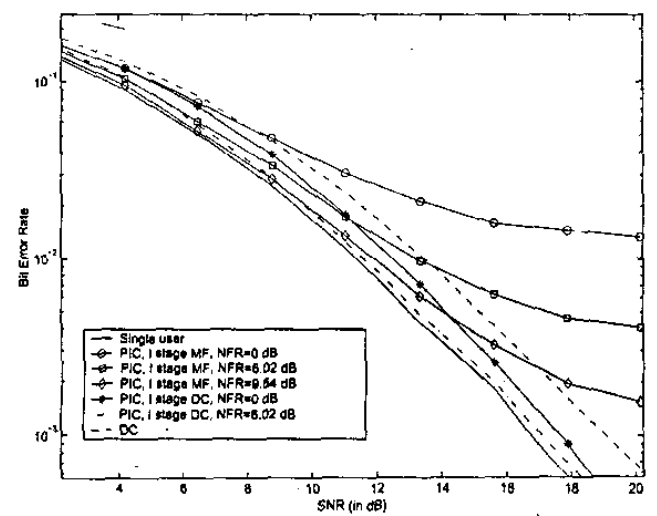

Fig. 4. BER performance of PIC for user 1 as a function of SNR in a 2-user space-time coded CDMA system. $M=2, \rho=0.8$. Perfect fade amplitude estimates.

correlation coefficient, $\rho$, considered are $0.6,0.7$ and 0.8 . We have assumed perfect knowledge of the channel matrices and the correlation matrix for the detection. The complex channel gains, $h_{i q}(1)$, in the channel matrix, $\mathbf{H}$, are taken to be complex Gaussian so that the fade amplitudes are Rayleigh distributed. We evaluate the near-far resistance capability of the SIC and PIC for different values of near-fat ratio ${ }^{1}$ (NFR).

Fig. 3 shows the BER performance plots for SIC with two different first stage detectors (namely, MF as the first stage and $D C$ as the first stage) at various values of near-far ratios (NFR $=0 \mathrm{~dB}, 6.02 \mathrm{~dB}$ and $9.54 \mathrm{~dB}$ ) for $\rho=0.8$. Perfect timing and fade amplitude estimates have been assumed in generating the plots in Fig. 3. From Fig. 3, it is observed that as the NFR increases the performance of SIC with MF as the first stage improves and moves closer to the single user performance, which can be explained as follows. When the NFR is high (the interfering user is much stronger than the desired

'We defin ne near-far ratio as $10 \log \frac{\sum_{p=1}^{M} \frac{A_{2 p}^{2}}{\sum_{p=1}^{M}} \cdot A_{1 p}^{2}}{A^{2}}$ assuming $E\left|h_{i q}(n)\right|^{2}=1$.
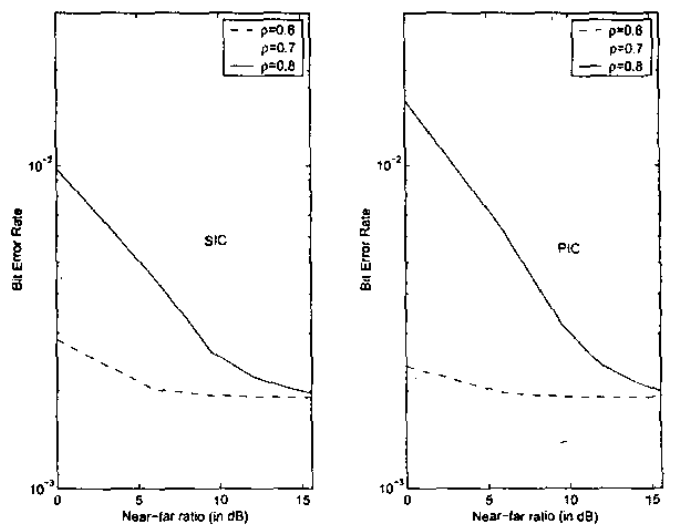

Fig. 5. BER performance of SIC/PIC for user 1 as a function of NFR in a 2-user space-time coded CDMA system for different values of $\rho . M=2$. $\mathrm{SNR}=15.62 \mathrm{~dB}$. Perfect fade amplitude estimates.

user), the reliability of the data estimate of the interfering user becomes very good, which, in turn, can result in accurate regeneration and cancellation of this interfering user's signal in the detection of the desired user in the subsequent stages. In our two-user case, the second user's data estimation (in the first stage) and cancellation (in the second stage) is effective and improves the performance of the first user for high NFR values. It is also seen that beyond an SNR of $10 \mathrm{~dB}$, if we use a more reliable first stage detector (e.g., DC), then even at low NFR ratios $(0 \mathrm{~dB}$ and $6.02 \mathrm{~dB}$ ) the performance of SIC approaches the single user performance. The BER performance of DC is also plotted to show that the SIC with DC as the first stage can outperform DC for SNRs beyond $10 \mathrm{~dB}$. In Fig. 4 , we present a similar performance comparison for the PIC detector. It is observed that the above discussions for SIC is equally valid for PIC with slight deviations in the quantitative aspect. We see that at low NFR, PIC with DC as the first stage outperforms the PIC with MF as the first stage over the range of SNRs. Also, PIC with DC as the first stage is better than the DC detector without IC.

The loss of near-far resistance at low NFR values is more clearly illustrated in Fig. 5, where the bit error tate performance of SIC and PIC (with MF as the first stage) are plotted as a function of NFR at an SNR of $15.62 \mathrm{~dB}$ for different values of $\rho$. It is observed that the loss is near-far resistance at low NFRs increases as the value of $\rho$ is increased. In Fig. 6 , the bit error rate performance of SIC and PIC (with MF and $\mathrm{DC}$ as first stage) are plotted for two values of SNR (15.62 dB and $13.34 \mathrm{~dB}$ ). All the detectors tend to perform similar when NFR is high. However, in the low NFR region, the SIC/PIC with MF as the first stage becomes less near-far resistant. The SIC/PIC with DC as the first stage retain the near-far resistance better even at low NFR values. In fact, in the low NFR region, the SIC/PIC with $\mathrm{DC}$ as the first stage performs better than SIC/PIC with MF as the first stage by an order of magnitude in BER. If a MF is preferred as the first stage because of its lesser complexity, then some amount of transmit power control will be preferred to take care of the near-far resistance 


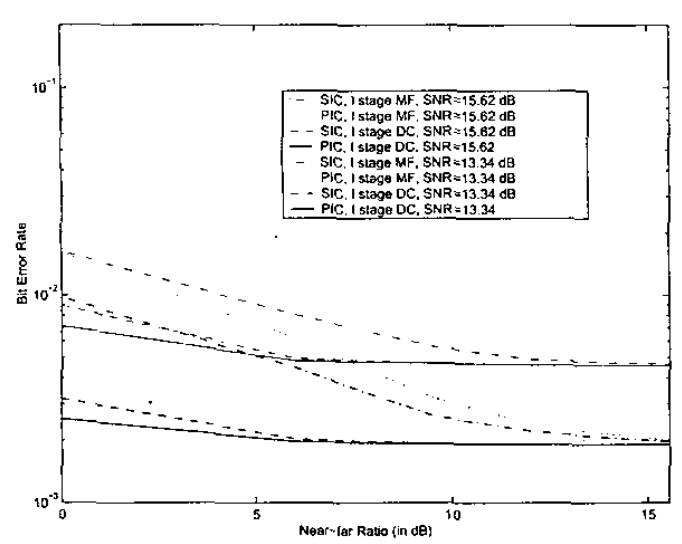

Fig. 6. BER performance of SIC/PIC for user 1 as a function of NFR in a 2-tiser space-time coded CDMA system. $M=2 . \rho=0.8$. Perfect fade amplitude estimates.

loss at low NFR conditions.

In Fig. 7, we illustrate the effect of inaccuracies in the fade amplitude estimates on the performance of SIC/PIC. We model the fade amplitude estimation error by perturbing the magnitude of the elements in the channel matrix $\mathbf{H}$ by a certain percentage of its true value and using this modified channel matrix for detection. Interestingly, the performance of SIC/PIC with MF as first stage, in the presence of $10 \%$ amplitude estimation errors, improves for increasing NFR values up to some NFR value (around $10 \mathrm{~dB}$ ), after which the performance starts degrading. It is thus observed that the SIC and PIC loose their near-far resistance both in the low and high NFR conditions, but for different reasons. In the low NFR region, it is due to the unreliable estimates of the interfering bits, whereas in the high NFR region it is due to imperfect fade amplitude estimates. This points out that in a practical scenario, some amount of transmit power control will be desirable to counter the effect of these performance losses in SIC/PIC.

\section{CONCLUSIONS}

We investigated the bit error performance of multistage interference cancellers (ICs) in a space-time coded CDMA system with multiple transmit antennas, under near-far conditions. Our performance results showed that a) when accurate fade amplitude and timing estimates are available, the ICs are less near-far resistant at low NFRs compared to that at high NFRs, due to the unreliability of the estimates of the interfering users' data in the initial stages of the IC at low NFRs, and b) in the high-NFR region, the near-far resistance is degraded due to inaccuracies in fade amplitude estimates. This indicates that some amount of transmit power control will be desirable in ICs to counter the loss of near-far resistance due to the above reasons.

\section{REFERENCES}

[1] V. Tarokh, N. Seshadri, and A. R. Calderbank, "Space-time codes for high data rate wireless communications: Performance criterion and code

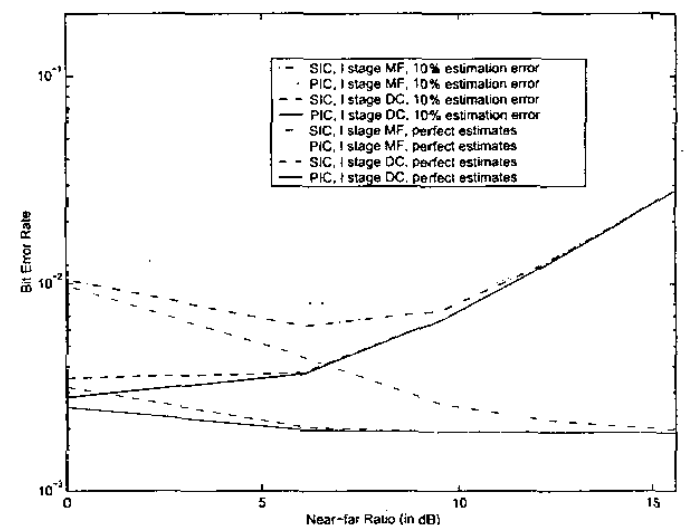

Fig. 7. BER performance of SIC/PIC for user 1 as a function of NFR in a 2-user space-time coded CDMA system in the presence of fade amplitule estimation errors. $M=2, \rho=0.8, \mathrm{SNR}=15.62 \mathrm{~dB}$.

construction," IEEE Trans. Inform. Theory, vol. 44, no. 2, pp. 744-765, March 1998.

[2] S. Alamouti, "A simple transmit diversity technique for wireless communications," IEEE Jl. Sel. Areas Commun., vol. 16, no. 8, pp. 14511458, October 1998.

[3] B. Hochwald, T. L. Marzetta, and C. B Papadias, "A transmitter diversity scheme for wideband CDMA systems based on space-time spreading." IEEE Jl. Sel. Areas Commun., vol. 19, no. 1, pp. 48-60, January 2001 .

[4] V. Tarokh, H. Jafarkhani, and A. R. Calderbank, "Space-time block codes from orthogonal designs," IEEE Trans. Inform. Theory, vol. 45, no. 5 , pp. 1456-1467, July 1999.

[5] S.Verdu, Multiuser Detection. Cambridge University Press: Cambridge, UK, 1998.

[6] A. F. Naguib, N. Seshadri and A. R. Calderbank, "Applications of spacetime block codes and interference suppression for high capacity and high data rate wireless systems," $32^{\text {nd }}$ Asilomar Conf. on signals, svstems and computers, pp. 1803-1810, 1998.

[7] H.Huang and $\mathrm{H}$. Viswanathan, "Multiple antennas and multiuser detection in high data rate CDMA systems," in Proc. IEEE VTC'2000, pp. $556-560,2000$.

[8] D. Reynolds, X. Wang, and H. V. Poor, "Blind adaptive space-time multiuser detection with multiple transmitter and receiver antennas," IEEE Trans. Sig. Proc., 50(6), pp. 1261-1276, June 2002.

[9] G. B Giannakis, Y. Hua, P. Stoica, and L. Tong, Signal Processing Advances in Wireless and Mobile Communications, Volume 2: Trends in Single-user and Multi-user systems, Prentice Hall PTR.

[10] M. M. Alam, "Performance of multiuser detectors for space-time coded CDMA," M.Sc (Engg) Thesis, Department of ECE, Indian Institute of Science, Bangalore, January 2003. 